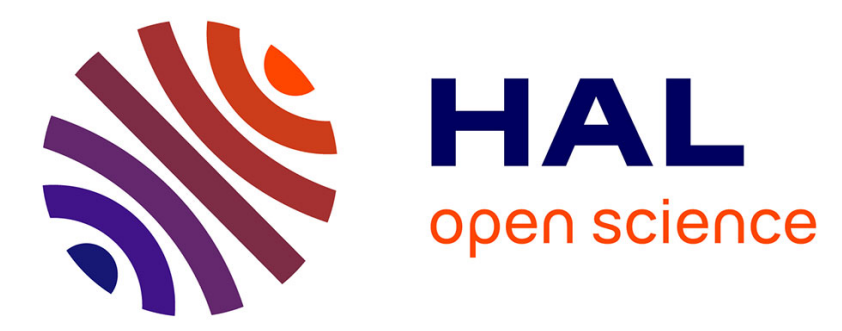

\title{
Motion Detector Placement Optimization in Smart Homes for Inhabitant Location Tracking
}

Maria Pia Fanti, Michele Roccotelli, Jean-Jacques Lesage, Gregory Faraut

\section{To cite this version:}

Maria Pia Fanti, Michele Roccotelli, Jean-Jacques Lesage, Gregory Faraut. Motion Detector Placement Optimization in Smart Homes for Inhabitant Location Tracking. ETFA'16: 21st IEEE Int. Conf. on Emerging Technologies and Factory Automation, Sep 2016, Berlin, Germany. hal-01357687

\section{HAL Id: hal-01357687 \\ https://hal.science/hal-01357687}

Submitted on 30 Aug 2016

HAL is a multi-disciplinary open access archive for the deposit and dissemination of scientific research documents, whether they are published or not. The documents may come from teaching and research institutions in France or abroad, or from public or private research centers.
L'archive ouverte pluridisciplinaire HAL, est destinée au dépôt et à la diffusion de documents scientifiques de niveau recherche, publiés ou non, émanant des établissements d'enseignement et de recherche français ou étrangers, des laboratoires publics ou privés. 


\section{Motion Detector Placement Optimization in Smart Homes for Inhabitant Location Tracking}

\author{
Maria Pia Fanti, Michele Roccotelli \\ Department of Electrical and Information Engineering \\ Politecnico di Bari \\ Bari, Italy \\ \{mariapia.fanti, michele.roccotelli\}@poliba.it
}

\author{
Jean-Jacques Lesage, Gregory Faraut \\ LURPA, ENS de Cachan \\ Université Paris-Sud, Univeristé Paris-Saclay \\ 94235 Cachan, France \\ \{jean-jacques.lesage, gregory.faraut $\} @$ lurpa.ens-cachan.fr
}

\begin{abstract}
The aim of the paper is to provide an optimal placement of sensors for inhabitant location tracking in smart homes, by using only motion detectors. In particular, motion detectors are binary sensors largely used in ambient assisted living applications because they are low cost, non-intrusive and privacy sensors. An approach to optimize the placement of motion detectors in a real home environment by adopting a twodimensional grid is presented. In this context, the real coverage area of a sensor is computed by considering the obstacles and respecting the specified coverage performance requirements. The optimization problem is formalized and solved as an Integer Linear Programming (ILP) problem and a case study is presented to show the efficacy of the proposed approach.
\end{abstract}

Keywords-Smart Home, Optimization, Motion Detector, Location Tracking.

\section{INTRODUCTION}

In recent years the development of smart home technologies allows helping people to live in more comfortable and safe environments. A smart home is equipped by sensors and actuators devices that can be remotely controlled. On the basis of the information given by the sensors, the actuators can be controlled for different purposes, e.g. to improve the comfort (heating, air conditioning and ventilation system, lights control, etc.) or to ensure the safety of inhabitants (fire or gas control, health problem detection, etc.) [1]. In this context, smart home technologies are largely used for Ambient Assisted Living (AAL) applications [2], [3], [10], [15]. AAL is a field of research that aims to improve the comfort and the safety of aged or disabled people living in their own dwelling [2]. It includes several applications such as the remote monitoring of patients having long term diseases, the control of smart equipments to support elderly people in their daily activities and the indoor location tracking of inhabitants [3]. Indoor location tracking is a meaningful evidence for numerous AAL activities. It consists in finding the location of inhabitants on the basis of the information given by different sensors. For instance, the knowledge of the real time location of inhabitants is helpful for the detection of health problems or to improve the comfort of the environment [2]. For these purposes, it is relevant to optimize the number and placement of sensors in smart homes in order to improve the location tracking performance [4]-[11].

This paper proposes an approach to optimize the placement of motion detectors for a given smart home topology by considering three aspects: i) the technology and the cost of sensors; ii) the obstacles that can limit the sensor detection; iii) the coverage requirements. The objective of the presented optimization problem is optimizing the placement of the sensors by minimizing the costs and satisfying the coverage requirements. This sensor placement optimization problem is formalized as an ILP problem by adopting a two dimension (2D) grid description of the flat. Moreover, the discussion of a case study shows the effectiveness of the proposed approach by considering different scenarios.

The paper is organized as follows: Section II analyzes the related works. Section III introduces the optimization approach for sensors placement and Section IV shows a case study by considering different scenarios for a given map of the flat. Finally Section V summarizes the conclusions and gives some perspectives for further works.

\section{RELATED WORKS}

The optimization of the sensor number and placement is a relevant topic for different fields of research. Many authors deal with this problem by adopting different approaches.

Feng. et al. [4] study the optimal design of an infrared motion sensing system for human motion localization in the context of human-following robots. In particular, they aim to find the optimal number and placement of Passive InfraRed (PIR) sensors in order to improve the localization performance. To this purpose, a multi-objective, mixed-integer-discretecontinuous optimization problem is presented and solved by using a divide and conquer based Genetic Algorithm method [4]. Moreover, Bishop et al. [5] aim to identify the optimal geometries for an arbitrary number of sensors and they show the influence of the sensor-target geometry on the potential localization performance by using analytical results and illustrative examples. In [6] Emmons et al. evaluate the optimal placement of binary sensors by providing several definitions for sensor coverage and spatial probability and by focusing on one dimension sensor arrays. A metric for measuring the overall utility of a given set of choices is presented that includes evaluation of the precision of measurements and coverage, and the associated costs. In addition, Zaho et al. [7] face the problem of finding the optimal sensor placement that can minimize the target tracking uncertainty by studying a control strategy that provides a flexible solution to autonomous optimal sensor deployment in 2D and 3D spaces. Differently, Farkas [8] investigates how to place wireless references ZigBee sensors to perceive the signal of at least three sensors in each point of a grid by minimizing the number of sensors. To this purpose a simulated annealing based algorithm to find the optimal number and placement of the reference sensors is proposed. Hiromori et al. [9] deal with the sensor placement optimization problem for 
multi-point pedestrian flow monitoring. To reach this goal, they propose an algorithm based on a Simulated Annealing approach and iteratively improve solutions to converge to near optimal solutions.

In the previously mentioned works, different methods to solve the problem of sensors placement optimization in a given space $(1 \mathrm{D}, 2 \mathrm{D}$ or $3 \mathrm{D})$ for location tracking purposes are developed but none of them is used to solve the problem of inhabitants location tracking in smart homes by considering a real environment. In this context, Gaddam et al. [10] present a framework to care elderly people in smart home based on wireless sensors. The selection of sensors is justified by a sensor selection architecture on the basis of the performance assessment perspectives. An evaluation module choices the appropriate sensor nodes on the basis of performance requirements for observability, fault detection, fault robustness and cost. Then, an optimization module searches through the solution space for the optimal sensor number deployment. Anyway, the authors do not show the applicability of the method on a real home environment. On the contrary, Vlasenko et al. [11] propose a hardware/software platform that aims to help an inhabitant in performing several daily activities within his/her home environment. The goal is to recognize the general activities in real time, without violating the privacy and develop a method for planning the optimal deployment of binary passive infrared (PIR) motion sensors. They aim to optimize the placement of sensors on the basis of the zones visitation frequency by considering only one inhabitant. In Danancher et al. [1], [2] a method that aims at the systematic construction of a Discrete Event System model for real-time location tracking of one or multiple inhabitants into a smart home is proposed. In addition, a location tracking algorithm as well as a procedure to evaluate the relevance of a given instrumentation for location tracking, are proposed. Several criteria are defined to evaluate $a$ priori the performance of the chosen instrumentation and to help the expert for manually improving it. In particular, one of these criteria measures the inability of the sensors network to detect the presence of inhabitants in certain zones of the flat (unlocationable zones are so defined). In fact, according to this criterion, a zone has to be covered by at least one sensor to allow to detect the presence of inhabitants. On the basis of the defined criteria, the performance of the location tracking algorithm is evaluated by an expert that can decide if a modification of sensors in term of type, number and placement is necessary.

In order to overcome the problem of manually modifying the sensor network, this paper proposes an extension of [1] and provides an automatic approach to optimize the sensor placement by guaranteeing the respect of the coverage requirements [2].

To reach this objective, we formulate the following assumptions:

i. only PIR motion detectors are used to respect the privacy [1], [2], [11];

ii. the living space is discretized by means of a twodimensional grid because only the localization of inhabitants in $2 \mathrm{D}$ is required for $\mathrm{AAL}$ purposes [11]-[14].

\section{SENSOR PLACEMENT OPTIMIZATION APPROACH}

In this section, we introduce an approach to optimize the placement of motion detectors by minimizing the costs, maximizing the overlapping of detection zones of sensors and respecting specific coverage requirements in a domestic environment.

In particular, a database of sensors and a map of the dwelling are necessary inputs for the optimization. Moreover, considering the given map, independent zones can be identified and for each zone and an ILP problem is defined, by customizing it on the basis of the zone coverage necessities. Then, each independent ILP problem is solved and the optimal solution for sensor placement is given for each zone of the flat.

The scheme of the presented optimization strategy is shown in Fig. 1.

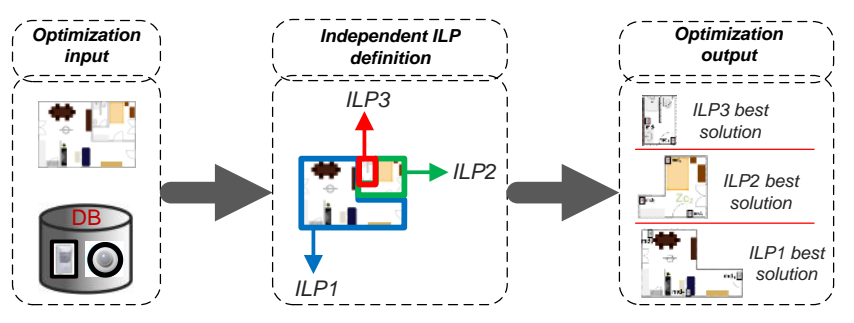

Fig. 1. Sensor placement optimization approach

\section{A. The Flat Map and Sensors Technology}

Let us consider an example of flat with the topology that is shown in Fig. 2.

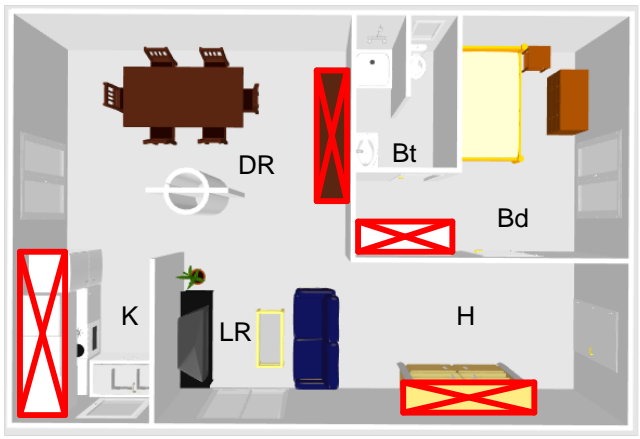

Fig. 2. The flat map

The flat is composed by several rooms: a hallway $(\mathrm{H})$, a living room (LR), a kitchen (K), a dining room (DR), a bedroom $(\mathrm{Bd})$ and a bathroom (Bt).

It is remarked that walls constitute obstacles for the sensing field of motion detectors. Indeed, the zone covered by motion detectors can be modified by the presence of internal walls and may generate zones not covered by the sensors. The sensing capacity of the considered motion detectors is characterized by a detection angle of $180^{\circ}$ and a detection range $\mathrm{R}$ (these values depend on the class of the PIR). Fig. 3(a) shows the detection zone including obstacles and Fig. 3(b) depicts the coverage zone after the $2 \mathrm{D}$ grid division of the zone. 
Moreover, internal walls, closets (signed by crosses in Fig. 2 ), open doors and windows may be considered as obstacles for the motion detectors.

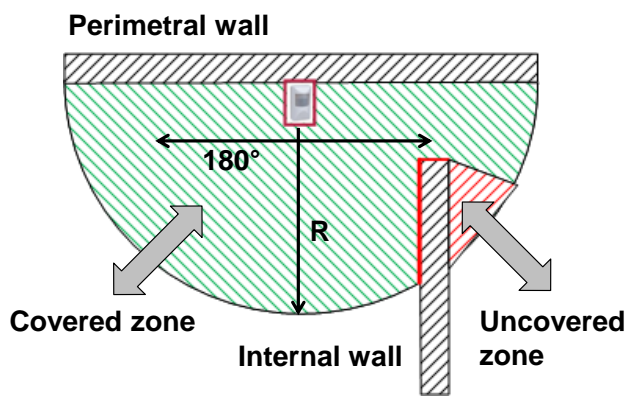

(a)

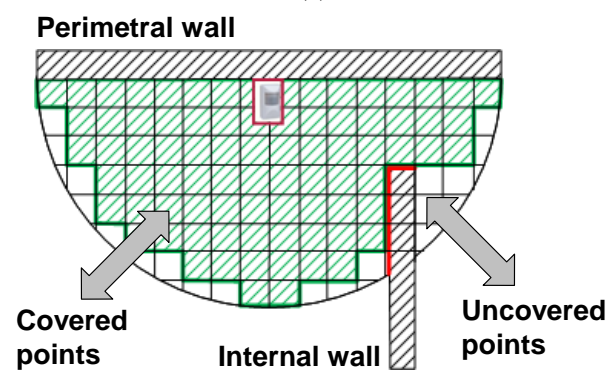

(b)

Fig. 3. Motion detectors coverage including obstacles: (a) coverage zone; (b) coverage points on the grid.

In the dwelling of Fig. 2, a set of internal walls identifies three closed zones (see Fig. 4): the hallway, the living room, the dining room, and the kitchen $\left(\mathrm{Zc}_{1}\right)$, the bedroom $\left(\mathrm{Zc}_{2}\right)$ and the bathroom $\left(Z_{3}\right)$. Since the three closed zones are limited by walls, each of them can be considered as an independent zone for sensor placements. For this reason, for each independent zone a sensor placement optimization problem can be defined.

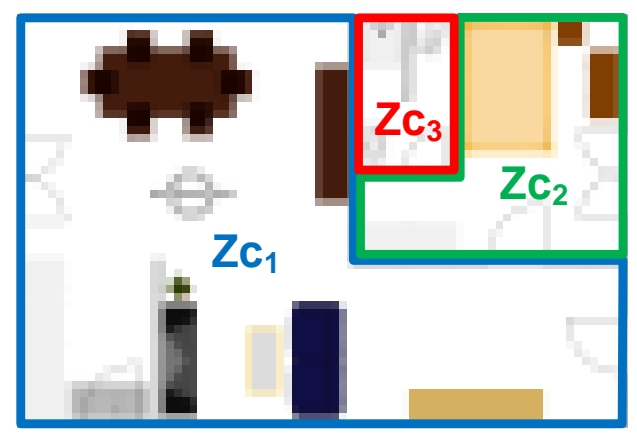

Fig. 4. The closed zones $\mathrm{Zc}_{1}, \mathrm{Zc}_{2}, \mathrm{Zc}_{3}$.

\section{B. The Integer Linear Programming Problem Definition}

In this subsection, we formalize the sensor placement optimization problem for each closed zone as an ILP problem.

In the proposed optimization framework, the objective is to optimize the placement of sensors, by minimizing costs and satisfying the zone coverage requirements. In order to formalize the problem, the following sets are defined:
$\mathcal{N}=\left\{i \in \mathbb{N}^{+} \mid i=1, \ldots, N\right\}$ is the set of grid points determined by dividing the zones in a $2 \mathrm{D}$ grid with suitable scales $g_{x}, g_{y}$. It is remarked that the designer can set the grid scales on the basis of the zone dimensions.

$\mathcal{P}=\left\{j \in \mathbb{N}^{+} \mid j=1, \ldots, P\right\}$ with $\mathcal{P} \subseteq \mathcal{N}$ is the set of grid points where the sensors can be placed;

$O=\{i \in \mathcal{N} \mid i$ is occupied by an obstacle $\}: O \subset \mathcal{N}$, set of points of the grid that are occupied by an obstacle;

$\mathcal{S}=\left\{m d_{k} \in \mathbb{N}^{+} \mid k=1, \ldots, S\right\}$ is the set of sensor types.

Example 1. Now in order to explain the 2D grid description of a closed room, a simple example is shown. Consider the rectangular zone $(A \times B)=(2 \times 1) m^{2}$ depicted in Fig. 5 where a grid scale of $g_{x}=g_{y}=g=1 \mathrm{~m}$ is applied. The resulting grid set $\mathcal{N}=\{i=1, \ldots, N\}$ is composed by $N=$ $(\lfloor A / g\rfloor+1) *(\lfloor B / g\rfloor+1)$ points and each point $i$ corresponding to the grid position $\left(x_{i}, y_{i}\right)$ is determined by the following formula:

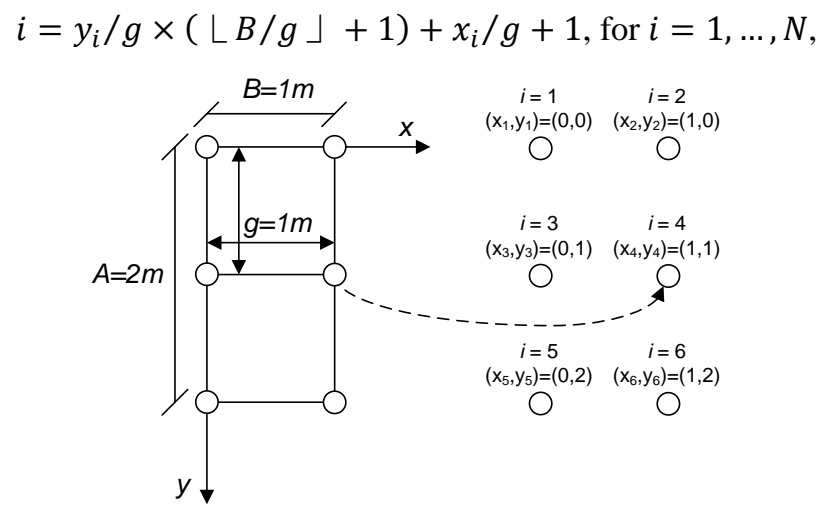

Fig. 5. The $2 \mathrm{D}$ grid description of a simple zone $(2 m \times 1 m)$.

where $\lfloor\alpha\rfloor$ denotes the floor function of $\alpha$, i.e., the largest integer not greater than the real number $\alpha$.

Moreover, each element $m d_{k} \in \mathcal{S}$ represents a motion detector type that is characterized by different technological and cost factors. In particular, the technological factor refers to the detection capacity of the sensor (detection angle and range), and the cost factor refers to the price of the sensor, including the cost for installation and maintenance. Hence, a cost is associated to each motion detector type $m d_{k} \in \mathcal{S}$ and is denoted by the element $c_{k}$ of vector $\boldsymbol{c} \in \mathbb{N}^{S}$.

In addition, the grid coverage is denoted by the following binary variables:

$g_{i j k}=\left\{\begin{array}{c}1, \quad \text { if sensor of type } k \text { that is placed } \\ \text { at point } j \text { covers point } i \\ 0, \quad \text { otherwise }\end{array}\right.$

for $i=1, \ldots, N, j=1, \ldots, P, k=1, \ldots, S$.

The decision variables are the binary variables $x_{j k}$ defined as follows:

$x_{j k}=\left\{\begin{array}{c}1, \quad \text { if sensor of type } k \text { is placed } \\ \text { at point } j \\ 0, \quad \text { otherwise }\end{array}\right.$ 
for $j=1, \ldots, P, k=1, \ldots, S$.

To specify the coverage of the grid points according to specific requirements and by excluding the obstacles, the following parameters are defined:

$a_{i} \in \mathbb{N}^{+}$if $i \in \mathcal{N} / O$, where $a_{i}$ represents the minimum number of sensors that have to cover the grid point $i$ that is not an obstacle;

$a_{i}=0$ if $i \in O$, i.e., $i$ is an obstacle.

The object of the problem is minimizing the costs of the sensors and maximizing the overlapping of the detection zones by assuring that all the grid points $i \in \mathcal{N} / O$ are covered according to the coverage requirements. It is remarked that the objective of maximizing the overlapping is necessary in order to allow as precise as possible determination of the inhabitants position.

Now, we define the following primary objective function expressing the minimization of total sensor cost:

$$
E=\min \sum_{j=1}^{P} \sum_{k=1}^{S} c_{k} x_{j k} .
$$

Moreover, we define the secondary objective function expressing the maximization of the overlapping of the zones covered by the sensors:

$$
E_{S}=\max \sum_{i=1}^{N} \sum_{j=1}^{P} \sum_{k=1}^{S} g_{i j k} x_{j k} .
$$

Then we optimize the two objectives in lexicographic order [17]: we first minimize $E$ and then we maximize $E_{S}$, while keeping $E$ at its minimum. The obtained mathematical formulation is the following ILP problem:

$$
\begin{aligned}
& \text { lexmin }\left[E, E_{S}\right] \\
& \text { subject to } \\
& \sum_{j=1}^{P} \sum_{k=1}^{S} g_{i j k} x_{j k} \geq a_{i}, \text { for } i=1, \ldots, N .
\end{aligned}
$$

The constraints $(3 \mathrm{~b})$ impose that the minimum number of sensors that have to cover the grid point $i \in \mathcal{N}$ is equal to $a_{i}$.

\section{CASE STUdy}

In this section a case study is presented to show the effectiveness of the optimization approach by considering three scenarios. To this purpose, we study the flat of Fig. 2 and 4 that has the following dimensions: the flat is of $6 \times 9=54 \mathrm{~m}^{2} ; \mathrm{Zc}_{1}$ is of $38 \mathrm{~m}^{2} ; \mathrm{Zc}_{2}$ is of $12.25 \mathrm{~m}^{2} ; \mathrm{Zc}_{3}$ is of $2.5 \times 1.5=3.75 \mathrm{~m}^{2}$.

Furthermore, we consider $g_{x}=g_{y}=g$ with different values of $g$ for the three closed zones, as reported in Table I. In

\begin{tabular}{|c|c|c|c|c|}
\hline & & & Closed Z & \\
\hline & & $Z c_{1}$ & $Z c_{2}$ & $Z c_{3}$ \\
\hline Grid sc & & $30 \mathrm{~cm}$ & $20 \mathrm{~cm}$ & $10 \mathrm{~cm}$ \\
\hline & $m d_{l}$ & & $\mathrm{R}_{1}=3 \mathrm{~m}$ & \\
\hline Sensor & $\boldsymbol{m d}_{2}$ & & $\mathrm{R}_{2}=6 \mathrm{~m}$ & \\
\hline & $\operatorname{md}_{3}$ & & $\mathrm{R}_{3}=10 \mathrm{~m}, \quad$ & \\
\hline
\end{tabular}
such a way we show that it is possible to choose the value of $g$ to modify the coverage accuracy.
TABLE I. GRID SCALES FOR THE THREE CLOSED ZONES AND CHARACTERISTICS OF THE CONSIDERED SENSORS

Moreover, the motion detector set is $\mathcal{S}=\left\{m d_{1}, m d_{2}, m d_{3}\right\}$ and includes three sensors that are characterized by the same detection angle $\left(180^{\circ}\right)$ with different ranges and costs as shown in Table I.

We assume that the placement of motion detectors is allowed only on the perimeter walls by excluding doors, windows, internal walls and closets (signed by crosses) as depicted in Fig. 6.

In the following subsections we solve the ILP problem (3(a),(b)) by considering three scenarios: i) in Scenario $1, Z_{c}$, $\mathrm{Zc}_{2}, \mathrm{Zc}_{3}$ are considered to show the coverage of the flat in presence of permanent obstacles (walls, closets); ii) in Scenario 2 , the zone $\mathrm{Zc}_{2}$ is studied in presence of open windows and open doors as additional obstacles for the sensor detection; iii) in Scenario 3, the ability of the methodology to take into account the sensor failures is studied by considering zone $\mathrm{Zc}_{1}$.

In each case the ILP problem is solved by using the solver GNU Linear Programming Kit [16] and an Intel-Core i52450M, CPU at $3.40 \mathrm{GHz}$, with 6GB RAM. The solution of the problems is obtained in at the most 570 seconds. Moreover, we remark that all the reported coordinates are expressed in meters and are computed as shown in Example 1 according to grid scales of Table I.

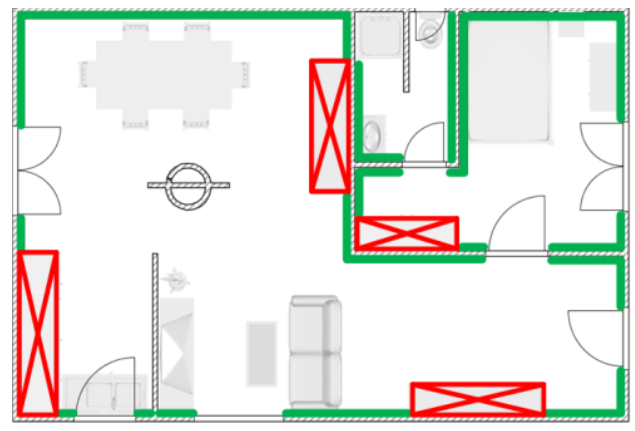

Fig. 6. The perimeter walls available for motion detectors placement

\section{A. Scenario 1}

In Scenario 1, for each independent zone, the objective is to optimize the placement of sensors by imposing that at least one sensor covers each zone not representing obstacles, i.e., $a_{i}=1$ if $i \in \mathcal{N} / O$ and $a_{i}=0$ if $i \in O$.

Assuming that the walls and the closets are obstacles and using the grid scales reported in Table I, the three zones are discretized as follows: 
$N=651, P=49$ for $\mathrm{Zc}_{1} ; N=441, P=35$ for $\mathrm{Zc}_{2} ; N=$ $375, P=49$ for $Z_{c_{3}}$. The reported values of $N$ and $P$ are also used in Scenario 2 and 3.

The results of the ILP solution for $\mathrm{Zc}_{1}$ are shown in Fig. 7. The optimal solution found has a cost of $135 €$ and is composed by three motion detectors: one sensor $m d_{1}$ is placed at grid position $\left(x_{465}, y_{465}\right)=(9,4.2)$ and two sensors $m d_{2}$ are placed at grid positions $\left(x_{5}, y_{5}\right)=(1.2,0)$ and $\left(x_{636}, y_{636}\right)=$ $(4.5,6)$, respectively.

Moreover, Fig. 8 depicts the result of the ILP solution for $\mathrm{Zc}_{2}$. The optimal solution is given by one sensor of type $m d_{2}$ placed at grid position $\left(x_{378}, y_{378}\right)=(4,3.4)$, that implies a cost of $50 €$.

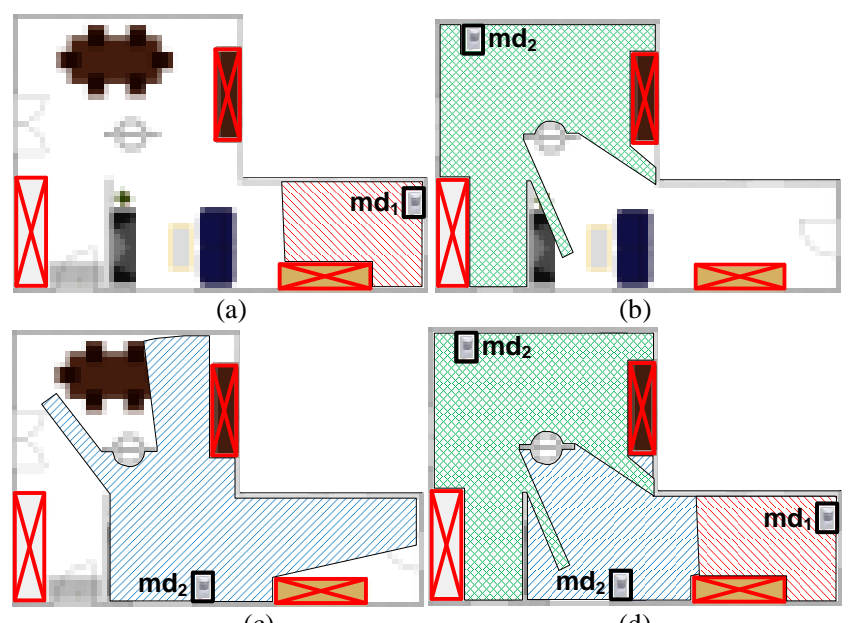

(c)

(d)

Fig. 7. ILP solution for $\mathrm{Zc}_{1}$ : (a) zone covered by $\mathrm{md}_{1}$ placed at grid point 465; (b) zone covered by $\mathrm{md}_{2}$ placed at grid point 5 ; (c) zone covered by $\mathrm{md}_{2}$ placed at grid point 636 ; (d) complete coverage of $\mathrm{Zc}_{1}$.

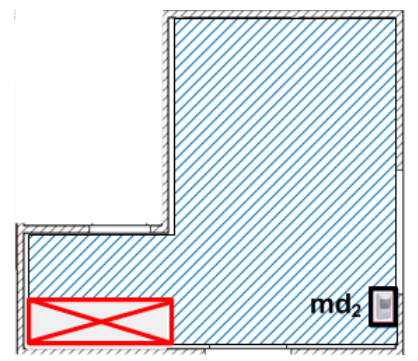

Fig. 8. The ILP solution for $\mathrm{Zc}_{2}$ : zone covered by one $\mathrm{md}_{2}$ placed at grid point 378.

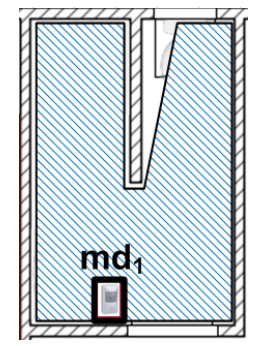

(a)

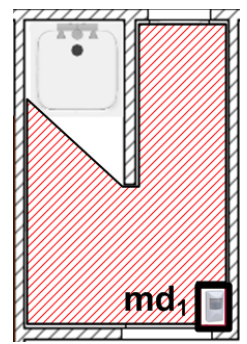

(b)
Fig. 9. The ILP solution for $\mathrm{Zc}_{3}$ : (a) zone covered by $m d_{1}$ placed at grid point 390; (b) zone covered by $m d_{1}$ placed at grid point 398.
Finally, the result of the ILP solution for $\mathrm{Zc}_{3}$ is shown in Fig. 9. The optimal solution is composed by two motion detectors of type $m d_{1}$ with a cost of $70 €$ : the first one is placed at grid point $390\left(x_{390}, y_{390}\right)=(0.5,2.4)$ and the second one is placed at grid point $398\left(x_{398}, y_{398}\right)=(1.4,0.4)$.

\section{B. Scenario 2}

In Scenario 2 we aim to cover zone $\mathrm{Zc}_{2}$ by considering the doors (fully opened) and the windows (fully opened) as additional obstacles and by keeping the coverage constraints imposed by Scenario 1 .

Therefore, we set $a_{i}=1$ if $i \in \mathcal{N} / O$ and $a_{i}=0$ if $i \in O$.

In this case, three sensors are necessary to cover zone $\mathrm{Zc}_{2}$ according to the coverage constraints with a cost of $135 €$ : one sensor $m d_{1}$ placed at grid position $\left(x_{436}, y_{436}\right)=(3,4)$ and two motion detectors of type $m d_{2}$ placed at grid positions $\left(x_{337}, y_{337}\right)=(0,3.2)$ and $10 \quad\left(x_{10}, y_{10}\right)=(1.8,0)$, respectively. In particular, the ILP solution is depicted in Fig. 10 where the coverage zones of the three sensors are respectively illustrated in Fig. 10(a), 10(b) and 10(c) while Fig. 10(d) shows the complete coverage of $\mathrm{Zc}_{2}$.

Comparing Fig. 8 and Fig. 10(d), it is evident that in Scenario 2 the number of motion detectors necessary to cover the grid according to the constraints is higher than in Scenario 1. This result is due to the increase of the number of obstacles, passing from Scenario 1 to Scenario 2. In particular, in the Scenario 1 , one sensor of type $m d_{2}$ is sufficient to appropriately cover $\mathrm{Zc}_{2}$, while in Scenario 2 three sensors (one $m d_{1}$ and two $m d_{2}$ ) are necessary. Although the ILP solution of Scenario 2 has an higher cost, it ensures location tracking even if the doors and windows are open.

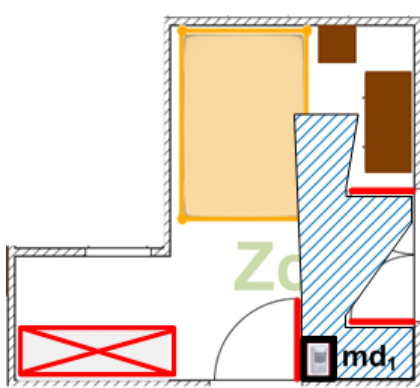

(a)

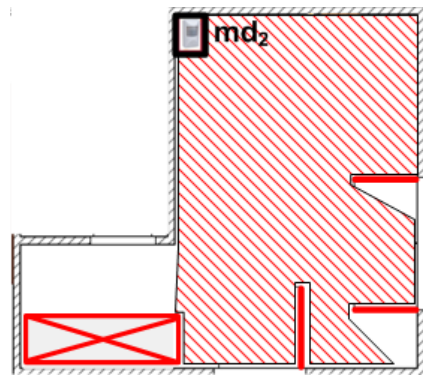

(c)

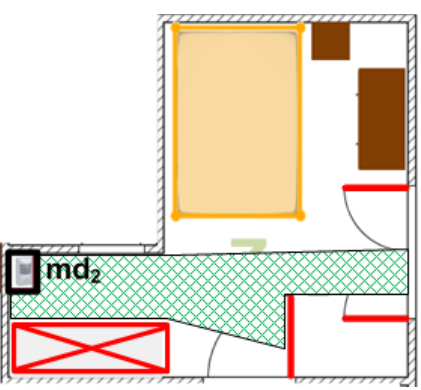

(b)

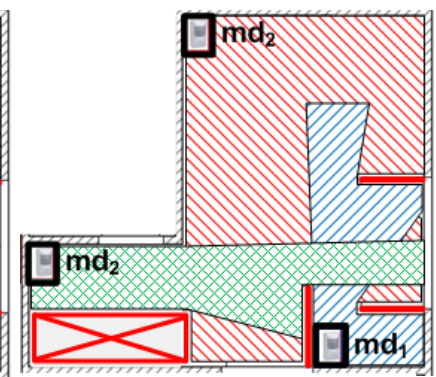

(d)
Fig. 10. $I L P$ solution for $\mathrm{Zc}_{2}$ : (a) zone covered by $m d_{1}$ sensor placed at grid point 436; (b) zone covered by $m d_{2}$ sensor placed at grid point 337; (c) zone covered by $m d_{2}$ sensor placed at grid point 10 ; (d) complete coverage of $\mathrm{Zc}_{2}$. 


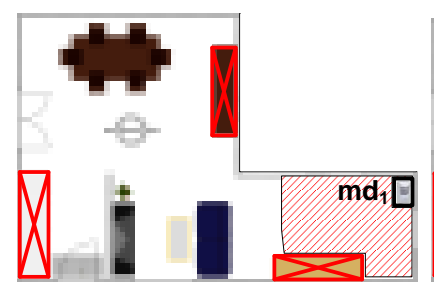

(a)

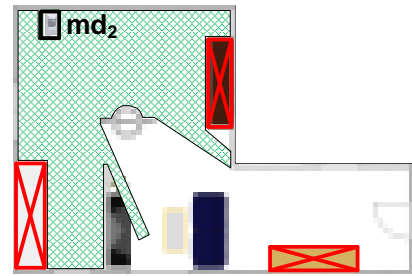

(c)

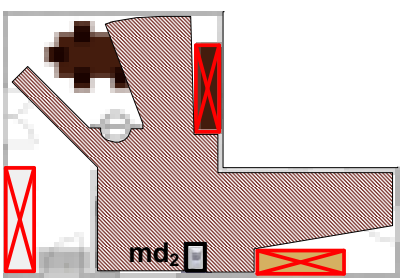

(e)

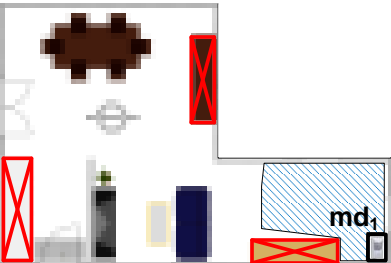

(b)

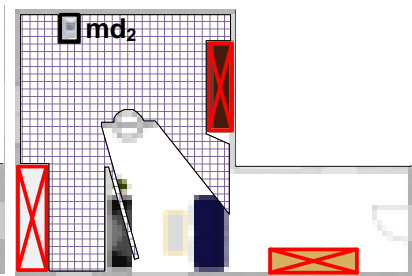

(d)

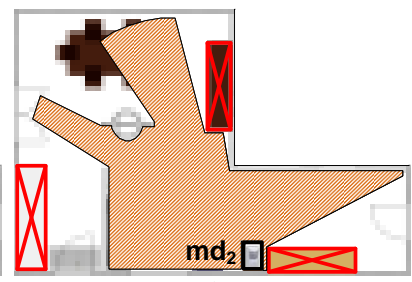

(f)
Fig. 11. ILP solution for $\mathrm{Zc}_{1}$ : (a) zone covered by $m d_{1}$ placed at grid point 465 ; (b) zone covered by $m d_{1}$ placed at grid point 620 ; (c) zone covered by $m d_{2}$ placed at grid point 4; (d) zone covered by $m d_{2}$ placed at grid point 5; (e) zone covered by $m d_{2}$ placed at grid point 636 ; (f) zone covered by $m d_{2}$ placed at grid point 637.

\section{Scenario 3}

In Scenario 3 the goal is to reinforce the coverage of zone $\mathrm{Zc}_{1}$ to be able to perform location tracking even in case of a sensor failure. In this case, the walls and the closets are considered as obstacles.

Therefore, we set $a_{i}=2$ if $i \in \mathcal{N} / O$ and $a_{i}=0$ if $i \in O$, to cover each point $i \in \mathcal{N} / O$ at least by two sensors.

The results of the ILP solution are shown in Fig. 11. In this case, the optimal solution is given by the combination of six sensors: two sensors of type $m d_{1}$ are placed at grid positions $\left(x_{465}, y_{465}\right)=(9,4.2) \quad$ and $\quad\left(x_{620}, y_{620}\right)=(9,5.7)$, respectively; four sensors of type $m d_{2}$ are placed at grid positions $\left(x_{4}, y_{4}\right)=(3,0),\left(x_{5}, y_{5}\right)=(4,0),\left(x_{636}, y_{636}\right)=(4.5,6)$ and $\left(x_{637}, y_{637}\right)=(4.8,6)$, respectively. In particular, the detection zones of the two $m d_{1}$ sensors are represented in Fig. 11(a)-(b) while Fig. 11(c)-(f) depict the detection zones of the four $m d_{2}$ sensors.

\section{CONCLUSIONS}

In this paper the sensor placement optimization problem in the context of inhabitants location tracking is solved by considering a real home environment and by using motion detectors only. In the proposed optimization approach, starting from a database of sensors and a map of the flat, an Integer Linear Programming problem is formulated in order to optimize the placement of sensors by minimizing the costs and respecting specific coverage requirements for each independent zone of the dwelling. The application to a case study shows that the proposed approach can provide different optimal solutions in different scenarios on the basis of the sensor costs and the requested coverage accuracy.

Future research will aim at extending the placement of motion detectors in all the flat and will consider other types of sensors in order to improve the accuracy of the grid coverage and the performance of the location tracking.

\section{REFERENCES}

[1] Danancher, M.; Lesage, J.-J.; Litz, L.; Faraut, G., "A Discrete event model for multiple inhabitants location tracking", in IEEE International Conference on," Automation Science and Engineering (CASE), 2013, pp.910-915, 17-20 Aug. 2013.

[2] Danancher, M.; Lesage, J.-J.; Litz, L.; Faraut, G., "Online Location Tracking of a Single Inhabitant Based on a State Estimator," in IEEE International Conference on Systems, Man, and Cybernetics (SMC), 2013, pp. 391-396, 13-16 Oct. 2013.

[3] Suryadevara, N.K.; Chia-Pang Chen; Mukhopadhyay, S.C.; Rayudu, R.K., "Ambient assisted living framework for elderly wellness determination through wireless sensor scalar data," in the Seventh International Conference on Sensing Technology (ICST), 2013, pp. 632639, 3-5 Dec. 2013.

[4] Feng, G.; Yang, Y.; Guo, X.; Wang G., "Optimal design of infrared motion sensing system using divide-and-conquer based genetic algorithm," in IEEE International Conference on Mechatronics and Automation (ICMA), pp.482-487, 4-7 Aug. 2013.

[5] Adrian N. Bishop, Barış Fidan, Brian D.O. Anderson, Kutluyıl Doğançay, Pubudu N. Pathirana, "Optimality analysis of sensor-target localization geometries", in Automatica, Volume 46, Issue 3, March 2010, pp. 479492.

[6] Emmons, S. P.; Kamangar, F, "Evaluating the optimal placement of binary sensors", in International Journal of Information Sciences \& Techniques, Vol. 3 Iss. 1, p1. January 2013.

[7] Zhao, S.; Chen, B.M.; Lee, T.H., "Optimal deployment of mobile sensors for target tracking in 2D and 3D spaces," in Journal of Automatica Sinica, IEEE/CAA, vol.1, no.1, pp. 24-30, Jan. 2014.

[8] Farkas, K., " Placement Optimization of Reference Sensors for Indoor Tracking", in Journal of applied sciences of Acta Polytechnica Hungarica, Vol. 12, Iss. 2, 2015.

[9] Hiromori, A.; Yamaguchi, H.; Higashino, T., "Sensor Placement Optimization Method for People Tracking," in Seventh International Conference on Next Generation Mobile Apps, Services and Technologies (NGMAST), pp. 62-67, 25-27 Sept. 2013.

[10] Gaddam, A.; Kaur, K.; Mukhopadhyay, S.C.; Gupta, G.S., "Selection and optimization of wireless sensors in a smart digital home for the elderly," in IEEE Sensors, pp.1382-1386, 25-28 Oct. 2009.

[11] Vlasenko, I.; Nikolaidis, I.; Stroulia, E., "The Smart-Condo: Optimizing Sensor Placement for Indoor Localization," in IEEE Transactions on Systems, Man, and Cybernetics: Systems, vol.45, no.3, pp.436-453, March 2015.

[12] Younis, M.; Akkaya, K., "Strategies and techniques for node placement in wireless sensor networks: A survey", Ad Hoc Networks, vol. 6, iss. 4, pp. 621-655. June 2008.

[13] Zou, Y.; Chakrabarty, K., "Uncertainty-aware and coverage-oriented deployment for sensor networks", in Journal of Parallel and Distributed Computing, vol. 64, Iss. 7, pp. 788-798, July 2004.

[14] Dhillon, S.S.; Chakrabarty, K.; Iyengar, S.S., "Sensor placement for grid coverage under imprecise detections," in Proc. of the Fifth International Conference on Information Fusion, vol.2, pp. 1581-1587, 8-11 July 2002.

[15] Kovacshazy, T.; Fodor, G., "New approach to passive infrared motion sensors signal processing for Ambient Assisted Living Applications," in 2012 IEEE International Conference on Instrumentation and Measurement Technology (I2MTC), pp.2521-2525, 13-16 May 2012.

[16] www.gnu.org/software/glpk.

[17] Ehrgott, M, "Multicriteria optimization," Springer Science \& Business Media. 2006. 